\title{
A Prototype Design for a Virtual Hospice and Initial Usability Study
}

\author{
Andrea Taylor \\ The Glasgow School of Art \\ Moray, UK \\ A.Taylor@gsa.ac.uk
}

\author{
Geoff Wilcock \\ openbrolly \\ Moray, UK \\ geoff.wilcock@openbrolly.com
}

\begin{abstract}
Hospice provides physical, social, emotional and spiritual care for people with life-shortening illness. Providing equitable access to services is becoming increasingly difficult for all hospices due to a rising number of people living longer with life-shortening illness and limited healthcare resources. Consequently, hospices are increasingly looking at ways of using technology to deliver services over a distance. This paper presents a prototype design for a web-based system ('virtual hospice') to improve access to services provided by Highland Hospice in the UK, and an initial usability study involving three elderly male patients. Participants completed most of the usability tasks, made positive comments, and would definitely or likely recommend the system to people who might benefit from it. The findings were translated into recommended changes to the virtual hospice, and may be helpful for other $\mathrm{HCl}$ designers and researchers working in this area.
\end{abstract}

Hospice; palliative care; virtual hospice; usability testing.

\section{INTRODUCTION}

Hospice and the related clinical speciality 'palliative care' describe an approach to care in which an interdisciplinary support framework is built around an individual living with life-shortening illness that can be used and directed by that individual to optimise their potential despite the underlying disease process. Services can include art and complementary therapies, bereavement and family support, chaplaincy and spiritual care, occupational therapy and physiotherapy, as well as nursing and medical care. Providing equitable access to services is becoming increasingly difficult for all hospices due to a rising number of people living longer with lifeshortening illness and limited healthcare resources. In response to this challenge, hospices are increasingly looking at ways of using technology to deliver services to patients living at home. This paper presents a prototype 'virtual hospice' in development by a hospice in the UK (Highland Hospice), and the results of an initial usability study.

Highland Hospice provides specialist palliative care in the Highlands of Scotland. The Hospice serves a population of 220,000 people in an area of 10,000 square miles, comprising the largest and most sparsely populated part of the UK with a mountainous terrain and limited transport infrastructure. The Hospice provides an in-patient unit and a range of services at its main building in the city of Inverness, and a day therapy centre in outlying areas. A specialist palliative care team supports healthcare professionals to provide palliative care in patients' homes, care homes and hospitals. However, most patients access services based within the Hospice building in Inverness and tend to live within a 30-mile radius-given the difficulty of travel for a population who are often physically frail. The primary aim of the virtual hospice, called 'Highland Virtual Hospice' (HVH), is to improve access to Highland Hospice services, particularly for those who have difficulty with access e.g. due to distance of travel, mobility problems or poor health. The groundwork for the HVH has been informed by substantial research with stakeholders (e.g. Taylor et al., 2013). This paper contributes to the important but small body of research within $\mathrm{HCl}$ on technology in hospice care. The main contribution is a novel prototype design for a virtual hospice to improve access to hospice care, and the findings of an initial usability study indicating the potential usefulness of the system, which may be helpful for other $\mathrm{HCl}$ researchers working in this area.

\section{HIGHLAND VIRTUAL HOSPICE}

The $\mathrm{HVH}$ is at a prototype stage of development and currently incorporates messaging, prescribed digital resources and video consultations. The system is being piloted with a small number of Highland Hospice patients, who are set up and shown how to use it by the Hospice; patients are loaned an iPad if 
they do not own a suitable device. Activity on the $\mathrm{HVH}$ is monitored by two medical secretaries during office hours. A secretary alerts a Hospice team member(s) to respond to incoming messages within 24 hours and to check on patients enrolled but not active on the $\mathrm{HVH}$. The Home page is the first page that patients see when they log in to the $\mathrm{HVH}$. The Home page includes: a message alert (new or no new messages); a slider with clickable profile images of Hospice team members that link to short biographies; and the main navigation menu with links to the main pages-Home and My messages.

\subsection{Messaging}

From the My messages page, patients can: read messages sent by members of the Hospice team; send a message to the Hospice and view all their sent messages; mark a message as a Favourite and view all messages marked as Favourites; and search for messages using a text string. Messages sent by members of the Hospice team may include links to prescribed digital resources for patients to engage with at home e.g. video clips of movement and physical exercises, audio clips of mindfulness exercises and PDF files of information sheets. Messages sent by patients are addressed to the Hospice team as a collective-this reflects the multidisciplinary team-based approach of hospice care and avoids messages being sent to individual team members who may not be available to respond. The messaging service improves patients' access to specialist advice on their particular situation or concern-from how to attain and preserve good symptom control and pain relief, to how to cope with emotional distress, develop a positive outlook and make necessary adjustments in their lives.

\subsubsection{Prescribed Digital Resources}

Digital resources are 'prescribed' by members of the Hospice team by selecting from a bespoke library. The library is populated with content by the Hospice by directly uploading a resource (video, audio, PDF file) or by specifying the URL for a resource. For each resource, the Hospice team adds metadata to aid search/retrieval when composing messages. The metadata includes specialism, e.g. physiotherapy and pharmacy, and keywords, e.g. anxiety management (one or more options may be selected from a pre-defined list), and short description (free text area).

\subsection{Video Consultations}

Zoom video conferencing software (Zoom, n.d.) is used for video consultations between patients and the Hospice. Currently, video consultations are mostly conducted by the physiotherapist in order to improve patients' motivation and confidence towards exercising, and to assess response. Physical deconditioning is a particular problem for those receiving hospice care and the consequences of reduced physical function are manifold. In particular, fatigue and unsafe mobility significantly impact an individual's ability to manage their condition at home. Video consultations are scheduled via messaging on the HVH. Currently, patients manually launch Zoom as a separate application on their computer/iPad to join a meeting, although planned development work includes better integration. Video consultations significantly improve patients' access to the physiotherapy service, as the Hospice employs a single physiotherapist who is based in Inverness with limited time to travel to patients' homes.

\section{RELATED WORK}

The Canadian Virtual Hospice is a well-established web-based platform that allows anyone in Canada (patients, families, professionals) to email questions to a team of palliative care experts. However, unlike the $\mathrm{HVH}$, responses are typically formulated as a single (i.e. one-off) communication as staff are not in a position to become directly involved in care (Canadian Virtual Hospice, n.d.). Other key features of the Canadian Virtual Hospice include articles and video clips on a range of topics and discussion forums. The HVH also disseminates resources such as articles and media clips, however these are prescribed for patients on an individual basis. Within $\mathrm{HCl}$, there is a small body of research on technology use in hospice care. For example, Ferguson et. al (2014) studied communication technology use during the final days of a person's life and provided opportunities and recommendations for design, particularly with respect to achieving comfort. However, the study focused on the interactions between hospice patients and their families and friends (caregivers), and the insights are derived from interviews with family and friends. As recommended by the authors, this study contributes accounts from patients themselves on technology use. Haque et al. (2014) developed and piloted a digital health system (e-ESAS) for rural palliative care patients in Bangladesh that included a patient module and a doctor module to record and monitor symptoms respectively. The pilot showed better communication between doctors and patients resulting in more timely interventions. However, little detail is provided on how the system was assessed for usability. This study contributes a further system for rural palliative care and a more detailed account of its usability testing.

\section{METHOD}

A usability testing method was used to evaluate the $\mathrm{HVH}$. Given that the plan was to conduct rounds of usability testing as part of an iterative design process, we followed the research-based guideline by usability expert Jakob Nielsen to test with no more than five users (Nielsen, 2000; Nielsen and 
Landauer, 1993). While the 'Magic Number 5' guideline is debated (e.g. Bevan et al., 2003) the strategy of running multiple small tests is accepted. Highland Hospice identified five potential participants among the pilot users of the $\mathrm{HVH}$, all of whom agreed to take part in the study (four males, one female). To preserve patient confidentiality, a new $\mathrm{HVH}$ account was created for the study and populated with sample messages by the Hospice. The usability testing was conducted in each participant's home and lasted approximately one hour. Each participant was asked to complete a series of tasks using the computer/iPad that they ordinarily access the HVH on, followed by a questionnaire. Participants were asked to verbalise their thoughts as they performed each task and completed the questionnaire (think aloud), which were captured using field notes and audio recordings. The results of the testing were translated into recommended changes to the $\mathrm{HVH}$.

\subsection{Tasks}

Participants were asked to complete the following tasks:

1. Read the message titled Leg exercises video. Play the video clip. Stop and close the video clip.

2. Read the message titled Relaxation script. Play the audio clip. Stop and close the audio clip.

3. Read the message titled Relaxation. Read, then close the information sheet (PDF file).

4. Send a message to the Hospice. Check that the message has been sent.

5. Mark a message from the Hospice as a Favourite. View all messages from the Hospice marked as a Favourite.

6. Use the search box to find the message from the Hospice titled Relaxation. Clear the search results.

7. Go back to the Home page. Find out more about members of the Hospice team.

8. Launch Zoom. Join a Zoom meeting.

\subsection{Post-Task Overall Questionnaire}

After the tasks, each participant was asked to complete the System Usability Scale (SUS) that can be used on small sample sizes (Brooke, 1996). The SUS provides a single number representing a composite measure of the overall usability of a system, ranging from 0 (negative) to 100 (positive). Each item is scored on a 5-point Likert Scale ranging from 'Strongly Disagree' to 'Strongly Agree'.

1. I think that I would like to use this system frequently.

2. I find the system unnecessarily complex.

3. I think the system is easy to use.
4. I think that I would need the support of a technical person to be able to use this system.

5. I find the various functions in this system are well integrated.

6. I think there is too much inconsistency in this system.

7. I would imagine that most people would learn to use this system very quickly.

8. I find the system very cumbersome to use.

9. I feel very confident using the system.

10. I needed to learn a lot of things before I could get going with this system.

In addition to the SUS, participants were asked to score the following question: 'I would recommend this system to people who might benefit from it'. The possible responses were: 'No, definitely not', 'No, I don't think so', 'Yes, I think so' and 'Yes, definitely'.

\section{RESULTS AND RECOMMENDATIONS}

Three elderly male patients, living 6-15 miles from the Highland Hospice building in Inverness and with a diagnosis of cancer, completed the usability testing (P1, P2, P3). All three lived at home: P1 and P2 lived with their wife and P3 lived alone following the death of his wife. Each patient experienced a particular challenge with distance of travel: travelling when on a course of chemotherapy (P1); moving around due to breathlessness and the use of a machine to take oxygen (P2); and not being able to drive anymore (P3). All three patients reported prior experience with computers, including email and the web, ranging from: 'knowing enough' and being 'a bit of a silver surfer' (P1); using technology 'not very often' (P2); and being a 'computer idiot' and finding 'computer language gobbledygook' (P3). To access the HVH, P1 used his iPad, P2 used his desktop computer and P3 used an iPad loaned by the Hospice. As pilot users, each participant had prior experience of the $\mathrm{HVH}$, although this was essentially limited to reading and sending messages. The other two patients who had agreed to participate became unable to for reasons that are not entirely clear.

\subsection{Key Usability Problems}

Participants completed the majority of the usability tasks, found the quality of the media clips to be very good, and considered the sample resources and Hospice team biographies to provide valuable information. A few tasks were not completed or completed with assistance, mostly due to:

1. A general lack of online instruction. For example, P2 and P3 struggled to remember how to mark a message as a Favourite and view messages marked as 
Favourites, and none could see how to access the Hospice team biographies.

2. Difficulties with the web browser itself. For example, P2 and P3 did not know how to stop the media clips as the browser default on their computer/iPad is to hide the control bar after a few seconds, made visible by moving the cursor over or tapping the media player area, and all participants experienced problems with the resources opening in a new browser window. For example, P1 and P3 did not know how to close the information sheet (PDF file) to get back to the original page: $\mathrm{P} 1$ relaunched the $\mathrm{HVH}$ and $\mathrm{P} 3$ could not see the tab to close it because the HVH opened in full screen on their iPad as configured by the Hospice.

\subsection{Post-Task Overall Questionnaire}

The SUS scores for participants were 67.5 (P1), 60 (P2) and 47.5 (P3): a score of 68 is considered average. Please refer to Brooke (1996) for how SUS scores are calculated. In addition to the SUS scores, P1 and P2 responded 'Yes, definitely' they would recommend the $\mathrm{HVH}$ to people who might benefit from it, and P3 responded 'Yes, I think so'. P1 commented 'I wasn't sure about it [HVH] at first as I'm a bit of a silver surfer, but it's extremely useful, particularly when travelling can be too much'. P2 similarly commented that the HVH is 'useful', has 'a positive and personal feel: this will help me, wow this is good' and would be particularly useful for people living distant from hospice facilities. P3 commented that he is 'gradually coming to terms with the iPad' and that the HVH 'gives one a certain feeling of security, you're in contact and you feel that you're being looked after, particularly if you live alone as I do, it's good to have contact'.

\subsection{Recommendations}

Based on the results of the usability testing, three easily implementable changes were recommended to the $\mathrm{HVH}$, to address the areas where participants experienced most difficulty:

1. Provide full(er) online instructions for use and improve the visibility of the interface functions and features

2. Provide training on basic computer/iPad skills and the web, as needed

3. Avoid opening new windows/tabs for the resources.

The first recommendation is a general principle for interaction design: instructions for use of a system should be visible or easily retrievable (recognition rather than recall). However, this study supports its relevance within the specific area of technology in hospice and palliative care. Hospice has a natural slant towards care for older people, given that most deaths occur among people who are older, and issues of memory are more severe compared to younger people. In particular, this is reflected in comments by P3 such as 'my memory isn't what it used to be'. An anticipated benefit of the $\mathrm{HVH}$, once fully developed, is that a reduction in time spent by the Hospice team travelling large distances to/from patients' homes and the main Hospice building in Inverness could result in more time for and/or an increased number of patient consultations. The recommendation to offer training on basic computer/iPad skills and the web could however result in time freed up by a virtual hospice being redirected back into training patients on how to use it-who, due to the nature of hospice care, might use it for a relatively short period of time. Morris et al. (2015) highlight the ways in which hospice volunteers occupy the space between the hospice and the community beyond its walls and the substantial scope to harness this volunteer link. It may be possible and desirable to upskill hospice volunteers, who play a key role in the functioning of hospices, to deliver any necessary training in patients' own homes or hospice facilities. The third recommendation supports other usability research with older people $(n=75)$ on the web, which proposed avoiding issues with multiple windows and as a rule of thumb only opening additional windows for PDF and other file types (Pernice et al., 2002). The results of this study suggest avoiding opening any new windows/tabs.

\subsection{Limitations}

The main limitation of this study is the small sample size of patients. Two of five participants dropped out once the study had started, reflecting the difficulty of engaging people receiving hospice care in research. However, it is important to stress that Nielsen has stated that three to four users is the optimal number for most usability studies, while five users should be planned for to allow for one or two dropouts (Bevan et al., 2003). The great overlap between observations of the three participants suggests that the study covered the main usability problems.

\section{CONCLUSION}

This paper contributes to the body of research within $\mathrm{HCl}$ on technology in hospice care. The results of the usability testing are preliminary, due to the early-stage design of the $\mathrm{HVH}$ and small sample size, but show promise that a virtual hospice can improve access to care. Participants completed most of the usability tasks, made positive comments, and would definitely or likely recommend the system to people who might benefit from it. Future work will include applying the 
recommendations of the study to the next iteration of the $\mathrm{HVH}$, and may explore extending the use of the system to patients' carers and providing a broader package of services.

Acknowledgments: Thank you to those who were involved for your time and support.

\section{REFERENCES}

Bevan, N., Barnum, C., Cockton, G., Nielsen, J., Spool, J. \& Wixon, D. (2003) The "magic number $5 "$ : is it enough for web testing? Proceedings of CHI 2003. Florida, 5-10 April 2003. New York: ACM. 698-699.

Brooke, J. (1996) SUS: A 'quick and dirty' usability scale. In Usability Evaluation in Industry, Jordan, P.W., Thomas, B., Weerdmeester, A. \& McClelland, I.L. (eds.). Taylor and Francis, London, UK, 4-7.

Canadian Virtual Hospice. http://www.virtualhospice.ca [20 March 2018].

Ferguson, R., Massimi, M., Crist, E. \& Moffatt, K. (2014) Craving, creating, and constructing comfort: insights and opportunities for technology in hospice. Proceedings of CSCW 2014. Maryland, 15-19 February 2014. New York: ACM. 1479-1490.

Haque, M., Kawsar, F., Adibuzzaman, M., Ahamed, S., Love, R., Dowla, R., Roe, D., Ferdousy, T. \& Selim, R. (2014) Findings of mobile based palliative care system: towards a generic framework for measuring QoL. Proceedings of Pervasive Health 2014. Germany, 20-23 May 2014. Belgium: ICST. 1-8.
Morris, S., Payne, S., Ockenden, N. \& Hill, M. (2015) Hospice volunteers: bridging the gap to the community? Health Soc Care Comm. 2015. doi:10.1111/hsc. 12232 .

Nielsen, J. (2000) Why you only need to test with 5 users. https://www.nngroup.com/articles/whyyou-only-need-to-test-with-5-users/ [20 March 2018].

Nielsen, J. \& Landauer, T. (1993) A mathematical model of the finding of usability problems. Proceedings of $\mathrm{CHI}$ 1993. Amsterdam, 24-29 April 1993. New York: ACM. 206-213.

Pernice, K., Estes, J. \& Nielsen, J. (2002) Senior citizens (ages 65 and older) on the web. http://www.nngroup.com/reports/seniors/ [20 March 2018].

Taylor, A., Lennox, J., Mort, A., Heaney, D., Muñoz, S-A., Currie, M., Hubbard, G., Steele, K. \& Keen, J. (2013) Developing hospice care over a distance in Highland Scotland: a knowledge exchange process. Proceedings of $\mathrm{CHI} 2013$. Paris, 27 April 27-2 May 2013. New York: ACM. 397-402.

Turner, C., Lewis, J. \& Nielsen, J. (2006) Determining usability test sample size. In International Encyclopedia of Ergonomics and Human Factors, Waldemar Karwowski (ed.). CRC Press, Boca Raton, FL, 3084-3088.

Zoom. Video conferencing and web conferencing. https://zoom.us/ [20 March 2018] 